\title{
Facile soft-chemistry synthesis of Co-Ru nanoalloys: influence of the composition on the catalytic activity for the acceptorless dehydrogenation of alcohols.
}

Brandon Azeredo, ${ }^{a}$ Tayssir Ben Ghzaiel, ${ }^{a}$ Ning Huang, ${ }^{a}$ Kamila Kaźmierczak, ${ }^{\text {b,c, }}$ Wenjie Shen, ${ }^{d}$ Guillaume Wang, ${ }^{e}$ Delphine Schaming, ${ }^{a}$ Patricia Beaunier, ${ }^{f}$ Philippe Decorse, ${ }^{a}$ Noémie Perret, ${ }^{b}$ Jennifer Peron, ${ }^{a}$ Marion Giraud, ${ }^{a}$ Carine Michel, ${ }^{c}$ Lorette Sicard, ${ }^{a,}{ }^{*}$ Jean-Yves Piquemal. ${ }^{a}{ }^{*}$

aUniversité de Paris, ITODYS UMR 7086, 15 rue Jean-Antoine de Baïf, 75013 Paris, France

bUniv Lyon, Université Claude Bernard Lyon 1, CNRS, UMR5256, IRCELYON, 2 avenue Albert Einstein, 69626, Villeurbanne, France.

'Univ Lyon, ENS de Lyon, CNRS UMR 5182, Université Claude Bernard Lyon 1, Laboratoire de Chimie, 69342, Lyon, France.

${ }^{d}$ State Key Laboratory of Catalysis, Dalian Institute of Chemical Physics, Chinese Academy of Sciences, Dalian, China.

${ }^{\mathrm{e}} \mathrm{MPQ}, \mathrm{CNRS}$, Université de Paris, F-75006 Paris, France.

fSorbonne Université, CNRS, UMR 7197, Laboratoire de Réactivité de Surface, Paris, 75005, France.

${ }^{\S}$ Present address: Total Energies One Tech Belgium, Zone Industrielle C, 7181 Feluy, Belgium.

*Corresponding authors : lorette.sicard@u-paris.fr, jean-yves.piquemal@u-paris.fr

Keywords: nanoparticles, alloys, cobalt, ruthenium, alcohols, catalytic acceptorless dehydrogenation of alcohols. 


\section{Abstract}

The synthesis of Co-Ru bimetallic nanoparticles was performed by co-reduction of Co(II) and $\mathrm{Ru}$ (III) salts in octan-1-ol, acting both as a solvent and a mild reducing agent. The metal composition was varied in the entire range, from pure Co to pure $\mathrm{Ru}$. Although $\mathrm{Co}$ and $\mathrm{Ru}$ are miscible in the bulk, the formation of nanoalloys is not straightforward and requires selecting carefully reaction parameters such as the nature of the solvent and that of the metal precursors. The formation of nanoalloys was unambiguously evidenced by HAADF STEM-EDX analyses. The particle size and the size dispersity were found to decrease with increasing $\mathrm{Ru}$ amount, yielding very small and monodisperse particles for the richest compositions in $\mathrm{Ru}$. The unsupported particles were tested for the acceptorless alcohol dehydrogenation using ( \pm )-octan-2-ol and octan-1-ol as model substrates. The results clearly show a synergetic effect since the bimetallic particles exhibit better performances than their monometallic counterparts. 


\section{Introduction}

Bimetallic nanoparticles (NPs) are fascinating objects that present a wide variety of parameters to play with such as the nature of the metals, composition, microstructure and morphological parameters. ${ }^{1,2}$ Depending on the mixing pattern of the two metals, different architectures can be generated such as phase-segregated Janus and core-shell particles or ordered (intermetallics) and random nanoalloys; ${ }^{3}$ these two last compounds tending to exhibit different physico-chemical properties. ${ }^{2}$ The possibility to finely adjust their electronic structure by judiciously selecting the two metals is very appealing, particularly for catalytic applications. ${ }^{4}$ In addition, mixing a noble metal with a non-noble one is a way to combine the generally high catalytic activity of the former and the superior selectivity of the latter. This very often results in enhanced catalytic performances for the bimetallic catalyst compared to its single-metal counterparts. ${ }^{4}$

For Fischer-Tropsch catalysis, ${ }^{5-7}$ it has been known for a long time that very small amounts of $\mathrm{Ru}$, used as a promotor, help to increase the reducibility of Co species. ${ }^{5,8}$ Recently, there has been an increasing interest in Co-Ru bimetallic catalysts for various reactions such as the hydrogenation of furfural to furfuryl alcohol, ${ }^{9}$ the decomposition of the $\mathrm{Li}_{2} \mathrm{CO}_{3}$ discharge product in $\mathrm{Li}-\mathrm{CO}_{2}$ batteries, ${ }^{10}$ the hydrolysis of aminoborane, ${ }^{11-13}$ the conversion of $\mathrm{CO}_{2}$ to methane ${ }^{14}$ or the oxygen ${ }^{15-17}$ and hydrogen ${ }^{12,13,16-20}$ evolution reactions. Bimetallic compounds are often generated through high temperature pyrolysis under neutral atmospheres and the active phase is stabilized thanks to interactions with a carbonaceous support such as carbon quantum dots, carbon nanotubes or graphene. ${ }^{16-18,20-}$ ${ }^{22}$ In some cases, the Co-Ru NPs are directly embedded in a carbon-based material, as a result of the preparation procedure, ensuring a better particle stabilization. ${ }^{12,17,18,20}$ However, because of the high temperature used in these syntheses, ill-defined, albeit isotropic particles are generally obtained with a broad size distribution.

On the contrary, methods aiming at preparing unsupported NPs by soft-chemistry routes are very scarce. Nevertheless, they are highly desirable because they would allow designing particles with specific structure, crystal habitus and surface state. These particles would also be more easily characterized than when deposited on a support and would present all their surface sites available for catalysis. Moreover, the influence of alloying on the properties of the material could be studied independently of the interactions with a 
support. Lastly, despite their size in the nanometer domain, the Co-Ru bimetallic NPs can be easily recovered due to their interesting magnetic properties. ${ }^{22,23}$ To the best of our knowledge, Zitoun et al. were the first to report the formation of unsupported $\mathrm{Co}_{\mathrm{x}} \mathrm{Ru}_{100-\mathrm{x}}$ particles prepared through organometallic pathway. ${ }^{23}$ The magnetic particles were stabilized with poly(vinylpyrrolidone) and characterized using HRTEM, WAXS and SQUID magnetometry. Although both structural and magnetic data were in favor of the formation of alloyed NPs, no definitive proof of an alloy could be obtained.

Recently, we reported the high activity and selectivity of unsupported Co NPs for the acceptorless dehydrogenation of alcohols. ${ }^{24}$ The catalysts were very efficient for the qualitative conversion of secondary alcohols while being inactive for primary ones. We also showed evidence of the presence of very small Ru NPs at the surface of the Co particles, that contributed to stabilize the catalytic activity, resulting in unmodified performances after three successive runs contrary to noble metal-free particles. ${ }^{25}$ These results were an incentive to shift from Ru-decorated Co NPs to Co-Ru bimetallic particles where the two metals would be homogeneously distributed inside the materials, i.e. to generate nanoalloys.

In this paper, we describe a simple soft chemistry procedure to prepare unsupported $\mathrm{Co}_{\mathrm{x}} \mathrm{Ru}_{100-\mathrm{x}}$ particles that rely on the use of commercially available metal precursors and a long-chain alcohol used as the solvent and a mild reducing agent. The nature of the Co and $\mathrm{Ru}$ precursors, as well as that of the alcohol were found to be keys to obtain alloys rather than phase-segregated materials. The magnetically recoverable nanoalloys were tested for a particularly important technological transformation: the acceptorless dehydrogenation of alcohols, using long-chain alcohols as model substrates. The results show that $\mathrm{Co}_{x} \mathrm{Ru}_{100-\mathrm{x}} \mathrm{NPs}$ are very efficient catalysts while a clear synergetic effect between the two metals can be observed.

\section{Experimental}

\subsection{Chemicals}

$\mathrm{Ru}(\mathrm{III})$ acetylacetonate (Alfa Aesar, $99 \%$ ), $\mathrm{RuCl}_{3} \cdot \mathrm{xH}_{2} \mathrm{O}$ (Sigma Aldrich, $99.9 \%$ ), $\mathrm{Co}(\mathrm{OAc})_{2} \cdot 4 \mathrm{H}_{2} \mathrm{O}$ (Sigma Aldrich, $\geq 98 \%$ ), tetrabutylammonium hexafluorophosphate (Sigma Aldrich, $\geq 99 \%$ ), octan-1-ol (AlfaAesar, > 99\%), ( \pm )-octan-2-ol (Alfa Aesar, > 98\%), n-decane (Carl Roth, > 99\%), 
butane-1,2-diol (Fluka, $98 \%$ ), ethanol (VWR, Normapur) and acetone (VWR, Normapur) were used without any further purification. Co(II) acetylacetonate hydrate (Sigma Aldrich, 97 $\%)$ was dried overnight in an oven at $60^{\circ} \mathrm{C}$ before use.

\subsection{Synthesis procedure}

The Ru and Co metal precursors were dissolved at room temperature in the selected solvent (octan-1-ol or butane-1,2-diol) with the desired $\mathrm{Co} / \mathrm{Ru}$ molar ratio and a total metallic concentration of $16.3 \mathrm{mmol} \mathrm{L}^{-1}$. The mixture was flushed with $\mathrm{Ar}$ in order to prevent metal oxidation. The solution was then heated up to the boiling point at a rate of $10^{\circ} \mathrm{C} \cdot \mathrm{min}^{-1}$. After 90 minutes under reflux, the solution was cooled down to room temperature. The nanoparticles were recovered by centrifugation at $20000 \mathrm{rpm}$ and washed once with absolute ethanol and once with acetone before being dried in an oven at $50{ }^{\circ} \mathrm{C}$ overnight. The samples are denoted $\mathrm{Co}_{x} \mathrm{Ru}_{100-\mathrm{x}}$ where $\mathrm{x}$ is in the range 0-100 and stands for the molar percentage of $\mathrm{Co}$ in the bimetallic sample.

\subsection{Characterization techniques}

Linear sweep voltammetry measurements were carried out at $25^{\circ} \mathrm{C}$ and at $60{ }^{\circ} \mathrm{C}$ in the presence of $\left[\mathrm{N}\left(\mathrm{C}_{4} \mathrm{H}_{9}\right)_{4}\right] \mathrm{PF}_{6}$ as the supporting electrolyte with a sweep rate of $5 \mathrm{mV} \cdot \mathrm{s}^{-1}$. The working, reference and auxiliary electrodes were respectively a Pt microelectrode of $25 \mu \mathrm{m}$ internal diameter, a saturated calomel electrode and a Pt wire.

X-ray diffraction patterns were collected using a Panalytical X'pert pro diffractometer equipped with a Co anode $(\lambda \mathrm{K} \alpha=1.7889 \AA)$ and a $X^{\prime}$ celerator detector. The refinements were carried out using MAUD software, ${ }^{26}$ based on the Rietveld method combined with Fourier analysis.

Thermogravimetric and thermodifferential analyses were performed using a Setaram Labsys Evo thermobalance under He from room temperature to $800{ }^{\circ} \mathrm{C}$ with a temperature ramp of $10{ }^{\circ} \mathrm{C} \mathrm{min}^{-1}$. Analyses were carried out under He atmosphere (Alphagaz 2 quality, $\mathrm{O}_{2} \leq 0.1$ ppm) in order to limit oxidation of the metal particles and avoid uncontrolled decomposition of the surface ligands. The thermobalance is coupled to an IR spectrometer and a GC-MS apparatus. Every minute, both an IR spectrum and a mass spectrum of the gas phase resulting from the pyrolysis of the powder are recorded. 
Routine transmission electron microscopy (TEM) and high-resolution microscopy (HRTEM) characterizations were performed using JEOL ARM 200F (equipped with a cold-field emission gun operated at $200 \mathrm{kV}$ and a CEOS aberration corrector of the objective lens) and JEOL JEM $2010\left(\mathrm{LaB}_{6}\right.$ filament and operating at $200 \mathrm{kV}$ ) transmission electron microscopes. The samples were prepared by evaporating a drop of a diluted suspension in ethanol on a carbon-coated copper grid. Mean particle sizes and standard deviations $(\sigma)$ were obtained through a statistic analysis by counting about 200 nanoparticles. High angle annular dark field scanning transmission electron microscopy (HAADF-STEM) coupled to EDX spectroscopy experiments were performed using a JEOL JEM 2100 Plus microscope (for linescan analyses) and a JEOL ARM 300F (for elemental mapping) microscope with a resolution of $0.063 \mathrm{~nm}$. For EDX analyses, the probe current was of $23 \mathrm{pA}$.

Elemental analyses of the powders were carried out by Energy Dispersive X-ray fluorescence (XRF) using an epsilon 3XL spectrometer from Panalytical equipped with a silver X-ray tube.

$X$-ray photoelectron spectroscopy (XPS) experiments were performed using a Thermo VG ESCALAB 250 system equipped with a micro-focused, monochromatic Al Kalpha X-ray source $(1486.6 \mathrm{eV})$ and a magnetic lens, which increases the electron acceptance angle and hence the sensitivity. The X-ray spot size was $650 \mu \mathrm{m}(15 \mathrm{kV}, 150 \mathrm{~W})$. The spectra were acquired in the constant analyzer energy mode with pass energies of 150 and $40 \mathrm{eV}$ for the general survey and the narrow scans, respectively. The data were processed using Avantage software. The peak binding energy positions were calibrated by setting the $C 1$ s component due to hydrocarbon contamination at $285 \mathrm{eV}$.

\subsection{Catalytic tests}

Catalytic tests were performed to assess the activity of $\mathrm{Co}_{x} \mathrm{Ru}_{100-x}$ particles in the dehydrogenation of ( \pm )-octan-2-ol and octan-1-ol in n-decane. They were conducted for $24 \mathrm{~h}$ at $145{ }^{\circ} \mathrm{C}$ using a given mass of catalyst to achieve an alcohol/[Co $\left.+\mathrm{Ru}\right]$ molar ratio of 100 (assuming the full metallic composition of the catalysts at the beginning), in $45 \mathrm{~mL}$ of liquids, with the alcohol concentration equal to $0.95 \mathrm{~mol} \mathrm{~L}^{-1}$, in a $100 \mathrm{~mL}$ semi-batch glass reactor with a constant flow of inert gases (90\% $\mathrm{Ar}$ and $10 \% \mathrm{~N}_{2}$, total flow $30 \mathrm{~mL} \mathrm{~min}{ }^{-1}$ ) and mechanical stirring (750 rpm). The reactor was coupled with a gas chromatograph (Shimadzu GC-2010, Supelco Carboxen-1010 PLOT column, thermal program: isotherm, $50{ }^{\circ} \mathrm{C}, \mathrm{Ar}$ carrier 
gas, TCD detector) to quantify the $\mathrm{H}_{2}$ production during the course of the experiment. Liquid aliquots of $0.50-0.75 \mathrm{~mL}$ were collected during the reaction and further analyzed by gas chromatography (Shimadzu, GC-2010, column ZB-FFAP, thermal program: gradient $40{ }^{\circ} \mathrm{C} \rightarrow$ $230{ }^{\circ} \mathrm{C}, 20^{\circ} \mathrm{C} \mathrm{min}^{-1}$, isothermal $230{ }^{\circ} \mathrm{C}, 10 \mathrm{~min}, \mathrm{~N}_{2}$ carrier gas, FID detector) to measure the concentration of the alcohol and of the corresponding carbonyl product in the reaction solution.

The conversion of the alcohol substrate, $X(\mathrm{ol})$, is calculated using: $X(\mathrm{ol}), \%=\frac{\mathrm{C}_{\mathrm{ol}}^{0}-\mathrm{Col}_{\mathrm{ol}}}{\mathrm{C}_{\mathrm{ol}}^{0}} \times 100$, where $C_{o l}$ is the concentration of the alcohol at a given time in liquid aliquots and $C_{o l}^{0}$ is the initial concentration of the alcohol. Selectivity towards the corresponding carbonyl compound, $S\left(\right.$ carbonyl), is defined as: $S$ (carbonyl), $\%=\frac{C_{\text {carbonyl }}}{C_{\text {carbonyl }}+\sum n \times C_{\text {by-products }}} \times 100$, where $\mathrm{C}_{\text {carbonyl }}$ is the concentration of the carbonyl compound (octan-2-one or octanal) at a given time in liquid aliquots, $\mathrm{n}$ is a stoichiometric coefficient and $\mathrm{C}_{\text {by-products }}$ is the concentration of the by-product(s) at a given time in liquid aliquots. The reaction yield in carbonyl product $\mathrm{Y}($ carbonyl) is given by: $\mathrm{Y}($ carbonyl), $\%=\mathrm{X}(\mathrm{ol}) \times \mathrm{S}($ carbonyl). The selectivity to octan-2-one and octanal are denoted respectively S(one) and S(al). In this work, S(one) was found to be close to $100 \%$, regardless the reaction time.

The yield in $H_{2}, Y\left(H_{2}\right)$, was also evaluated following: $Y\left(H_{2}\right), \%=\frac{n_{H_{2}}^{\text {exp }}}{n_{H_{2}}^{\text {theo }}} \times 100$, where $n_{H_{2}}^{\text {exp }}$ is the accumulated amount of $\mathrm{H}_{2}$ produced after a given time of reaction and $\mathrm{n}_{\mathrm{H}_{2}}^{\text {theo }}$ is the theoretical amount of $\mathrm{H}_{2}$ possibly produced during alcohol dehydrogenation, based on the initial amount of alcohol.

Finally, the turnover number, TON, is calculated as: $T O N=\frac{n_{\text {converted alcohol }}}{n_{\text {surface metal atoms }}}$ where $n_{\text {converted alcohol }}$ is the amount of alcohol converted into product(s) during the reaction, based on alcohol concentration at the beginning and end of the reaction and $n_{\text {surface metal atoms }}$ is the amount of surface $\mathrm{Co}$ and Ru atoms (first layer of metal) in the catalyst sample used in the reaction. This number was evaluated taking into account i) the composition of the nano-catalyst and ii) that the exposed facets exhibit different amounts of $\mathrm{Co}$ and $\mathrm{Ru}$ atoms per surface unit (see SI). The absolute error on TON is estimated to be $\pm 75 \mathrm{~mol} \cdot \mathrm{mol}^{-1}$. We also assumed a statistical distribution of the two metals into the particle. 


\section{Results and discussion}

In this work, bimetallic particles with the $\mathrm{Co}_{x} \mathrm{Ru}_{100-x}$ composition are prepared by a softchemistry route using either a diol (butane-1,2-diol) or a mono-alcohol (octan-1-ol), both acting as the solvent and the reducing agent. Even if $\mathrm{Ru}$ and Co are miscible elements over the entire composition range, ${ }^{27}$ demixing or formation of hetero-nanostructures such as Janus particles or core-shell particles can be observed if the reaction conditions are not finely controlled. Although standard electrode potentials are given at $25{ }^{\circ} \mathrm{C}$ for aqueous solutions, they can serve as a guide for assessing the relative reducibility of the metal salts used. $^{28}$ The large difference in reduction potentials $\left(E^{0}\left(\mathrm{Ru}^{3+} / \mathrm{Ru}\right)=0.45 \mathrm{~V}\right.$ vs. SHE and $\mathrm{E}^{0}\left(\mathrm{Co}^{2+} / \mathrm{Co}\right)=-0.28 \mathrm{~V}$ vs. SHE $)^{2}$ is clearly not in favor of a concomitant reduction of the two metal ions. Moreover, Ru and Co display very different cohesive energies and melting point values, precluding easy formation of the alloy. ${ }^{2}$ Consequently, the reduction kinetics of the two precursors have to be adjusted to allow the generation of the desired alloy. Key parameters that can be varied to reach this objective are the nature of the metal precursor and/or of the solvent. Several syntheses have thus been carried out with different metal precursors $\left(\mathrm{RuCl}_{3} \cdot \mathrm{xH}_{2} \mathrm{O}\right.$ and $\mathrm{Ru}(\mathrm{acac})_{3}$ for $\mathrm{Ru}(\mathrm{III})$ and $\mathrm{Co}(\mathrm{OAc})_{2} \cdot 4 \mathrm{H}_{2} \mathrm{O}$ and $\mathrm{Co}(\mathrm{acac})_{2}$ for $\left.\mathrm{Co}(\mathrm{II})\right)$ and with two solvents (octan-1-ol and butane-1,2-diol) displaying different reduction abilities in order to define the best conditions favoring the formation of an alloy. The influence of the composition ( $\mathrm{Co} / \mathrm{Ru}$ molar ratio) was also evaluated. First, the results obtained with the $\mathrm{Co}_{80} \mathrm{Ru}_{20}$ composition will be described, before reporting the results obtained with other compositions in a second step.

Among all the possible combinations (see SI, Sigure S1), it is only with the acac precursors in octan-1-ol that well-defined nanoalloys are generated (see Figure 1). Notably, the use of butane-1,2-diol (BD) instead of octan-1-ol, while keeping all other conditions identical, leads to the formation of particles that settle rapidly over time. A TEM analysis shows that large particles are obtained together with tiny NPs (see Figure S1, SI). Pure Co is detected by XRD while a broad peak centered at about $50^{\circ}$ is attributed to Ru particles corresponding to very small coherent diffraction domains (Figure S1, SI). To sum up, in BD, there is neither formation of a homogeneous alloy nor a control of the mean particle size. These results emphasize the role of the solvent which is also the reducing agent (as well as a 
possible ligand for the metal precursor and/or for the particle surface). It will be discussed later in this paper.

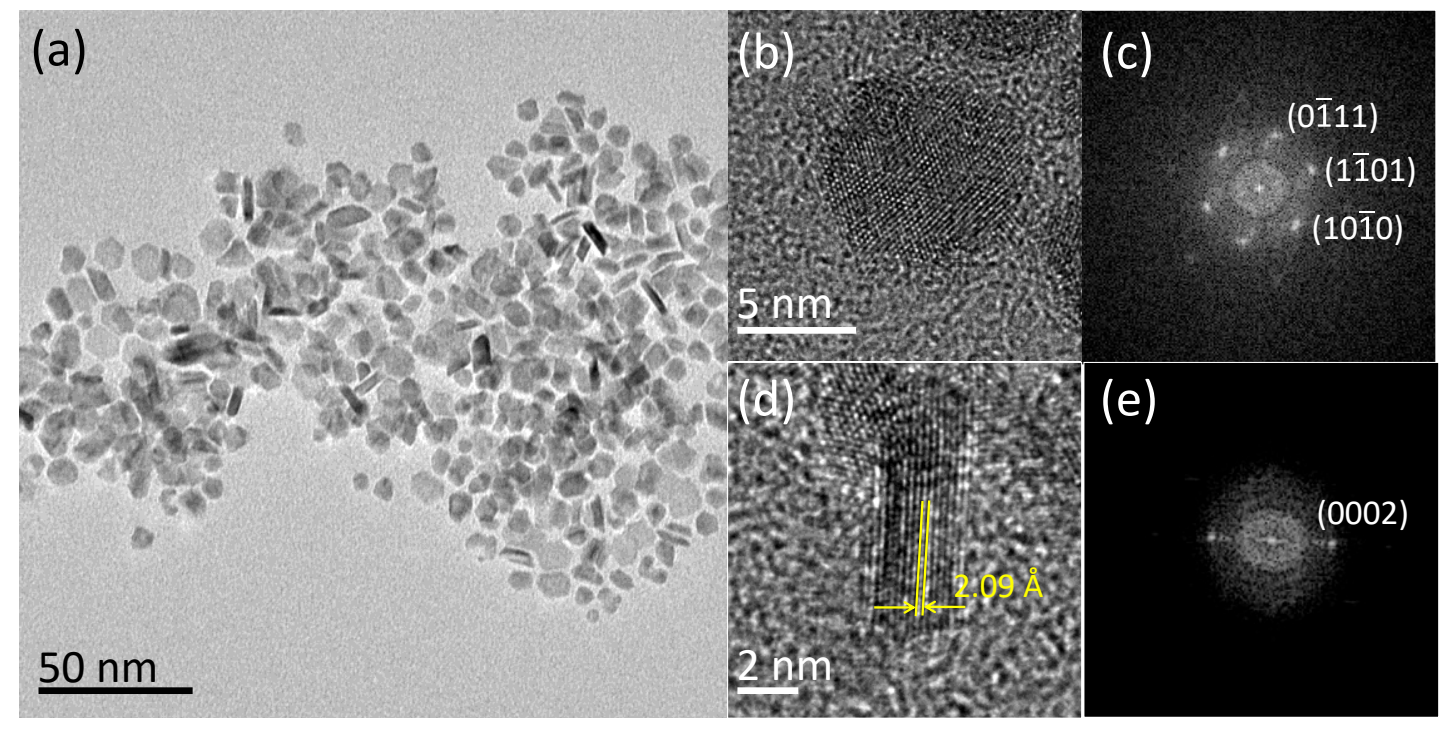

Figure 1. (a) TEM micrograph of the $\mathrm{CO}_{80} \mathrm{Ru}_{20}$ sample; (b) HRTEM image of a single NP viewed along the $[1 \overline{2} 1 \overline{3}]$ axis of the hexagonal system; (c) corresponding FFT of image b; (d) HRTEM image of a single NP viewed perpendicular to the $c$ axis of the hexagonal system and (e) corresponding FFT of image $d$.

$\mathrm{CO}_{80} \mathrm{Ru}_{20}$ powders are first analyzed by TEM (see Figure 1 and Figure S2). They correspond to platelets with a mean size of $6.6 \mathrm{~nm}(\sigma=1.4 \mathrm{~nm})$ while the platelet thickness is $2.5 \mathrm{~nm}(\sigma=0.4 \mathrm{~nm})$. HRTEM studies show that the particles are very well crystallized. The interplanar spacing $d_{\text {hkil }}$ of the (0002) lattice plane measured on the FFT image (Fig. 1e) is equal to $0.209 \mathrm{~nm}$. This value lies between those of pure hcp Co and hcp Ru (Co: $d_{0002}=$ $0.204 \mathrm{~nm}, \mathrm{ICDD} \#$ 98-004-4990 and Ru: $\mathrm{d}_{0002}=0.214 \mathrm{~nm}, \mathrm{ICDD} \#$ 98-004-3710). Assuming a Vegard's law, the composition of the particle is found to be $\mathrm{CO}_{50} \mathrm{Ru}_{50}$. XRD indeed reveals that the sample is actually a mixture of two hexagonal phases: one with the $\mathrm{CO}_{50} \mathrm{Ru}_{50}$ composition and another that was found to be $\mathrm{Co}_{86} \mathrm{Ru}_{14}$ thanks to a Rietveld analysis using the Maud software ${ }^{26}$ (see Figure 2a). Note that the global composition is however in very good agreement with the nominal composition $\left(\mathrm{CO}_{80} \mathrm{Ru}_{20}\right)$ as evidenced by XRF and XPS (vide infra). These results are confirmed by STEM-EDX analyses that provide invaluable high spatial resolution compositional information on alloy homogeneity and phase segregation effects within individual nanoparticles (Figure 2). 
(a)

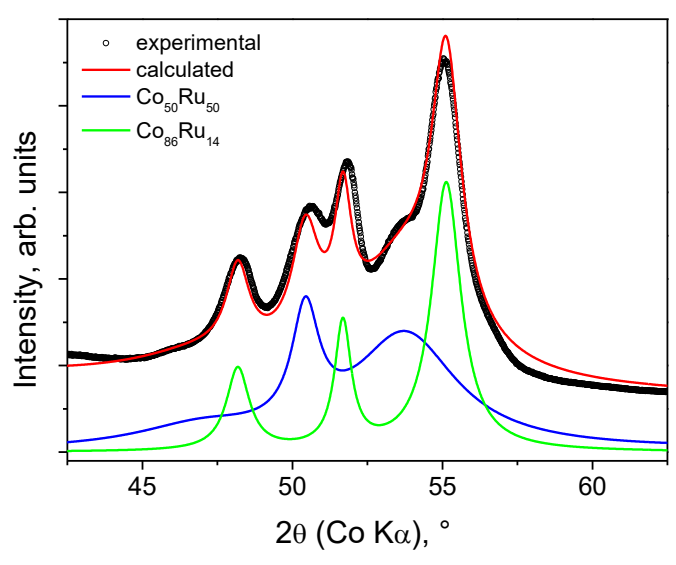

(b)
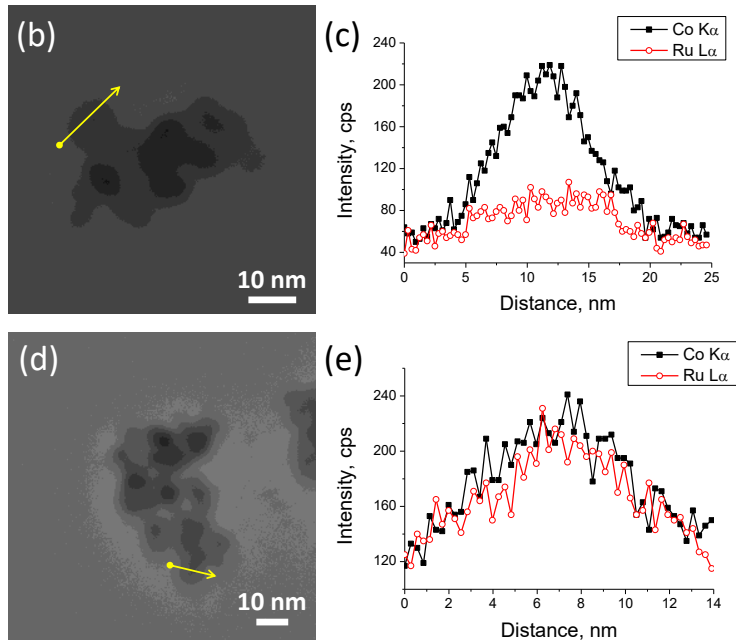

$\rightarrow$ e) $\rightarrow$ -

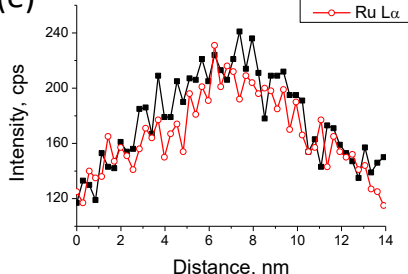

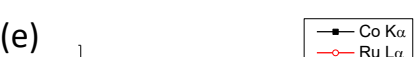

Figure 2. (a) X-ray diffraction pattern of the $\mathrm{Co}_{80} \mathrm{Ru}_{20}$ sample prepared in octan-1-ol, (b,d) STEM images of $\mathrm{CO}_{80} \mathrm{Ru}_{20} \mathrm{NPs}$ and $(c, e)$ corresponding EDX line profiles. The EDX analyses are performed along the yellow arrows indicated in $(b, d)$.

The elemental line profiles displayed in Figure 2 show that Co and $\mathrm{Ru}$ are homogeneously distributed in a single NP. This is also evidenced by STEM-HAADF coupled to EDX (see below). However, the Co/Ru molar ratio differs significantly from one NP to another, showing two populations, one nearly equimolar in $\mathrm{Co}$ and $\mathrm{Ru}$ and another one richer in Co. This is commonly encountered for alloys at the nanometer scale, prepared either through physical or chemical methods. ${ }^{29-31}$

$\mathrm{Co}_{x} \mathrm{Ru}_{100-x}$ particles with other compositions can also be prepared with this simple synthetic strategy. Figure 3 gathers TEM images for $\mathrm{Co}_{95} \mathrm{Ru}_{5}, \mathrm{Co}_{50} \mathrm{Ru}_{50}, \mathrm{Co}_{20} \mathrm{Ru}_{80}$ as well as pure $\mathrm{Co}$ and $\mathrm{Ru}$ samples for comparison purposes. Without $\mathrm{Ru}$ in the system, large Co aggregates are recovered which are comprised of ill-defined particles having sizes of a few tens of $\mathrm{nm}$. Increasing the $\mathrm{Ru}$ content leads to a dramatic decrease of the mean particle diameter together with an improvement of the size dispersity characterized by a marked decrease in the standard deviation (Figure $3 \mathrm{~g}$ and Figure $\mathrm{S} 2, \mathrm{SI}$ ). It is noteworthy to precise that the mean size given for the $\mathrm{Co}_{95} \mathrm{Ru}_{5}$ composition corresponds actually to that of the core since for this material, there is phase segregation with a Co-rich core decorated with Ru particles (vide infra and Figure 4). The $\mathrm{CO}_{50} \mathrm{Ru}_{50}$ and $\mathrm{CO}_{20} \mathrm{Ru}_{80}$ particles are very well defined and correspond to nanoplatelets exhibiting nearly the same mean size, respectively $3.91 \mathrm{~nm}$ $(\sigma=0.36 \mathrm{~nm})$ and $3.76 \mathrm{~nm}(\sigma=0.44 \mathrm{~nm})$. Finally, with pure $\mathrm{Ru}$, nanoflowers with a few tens 
of nanometers are recovered. These nanoflowers correspond to polycrystalline aggregates of tiny Ru NPs with a mean size of a few $\mathrm{nm}$. This peculiar morphology has already been described for $\mathrm{Ru}$ particles prepared via the organometallic pathway in alcohols or in alcohol/THF mixtures. ${ }^{32}$ As revealed by HRTEM studies, and similarly to $\mathrm{Co}_{80} \mathrm{Ru}_{20}, \mathrm{Co}_{50} \mathrm{Ru}_{50}$ and $\mathrm{Co}_{20} \mathrm{Ru}_{80}$ NPs crystallize with the hcp structure (see Figure $3 \mathrm{~d}$ and e).
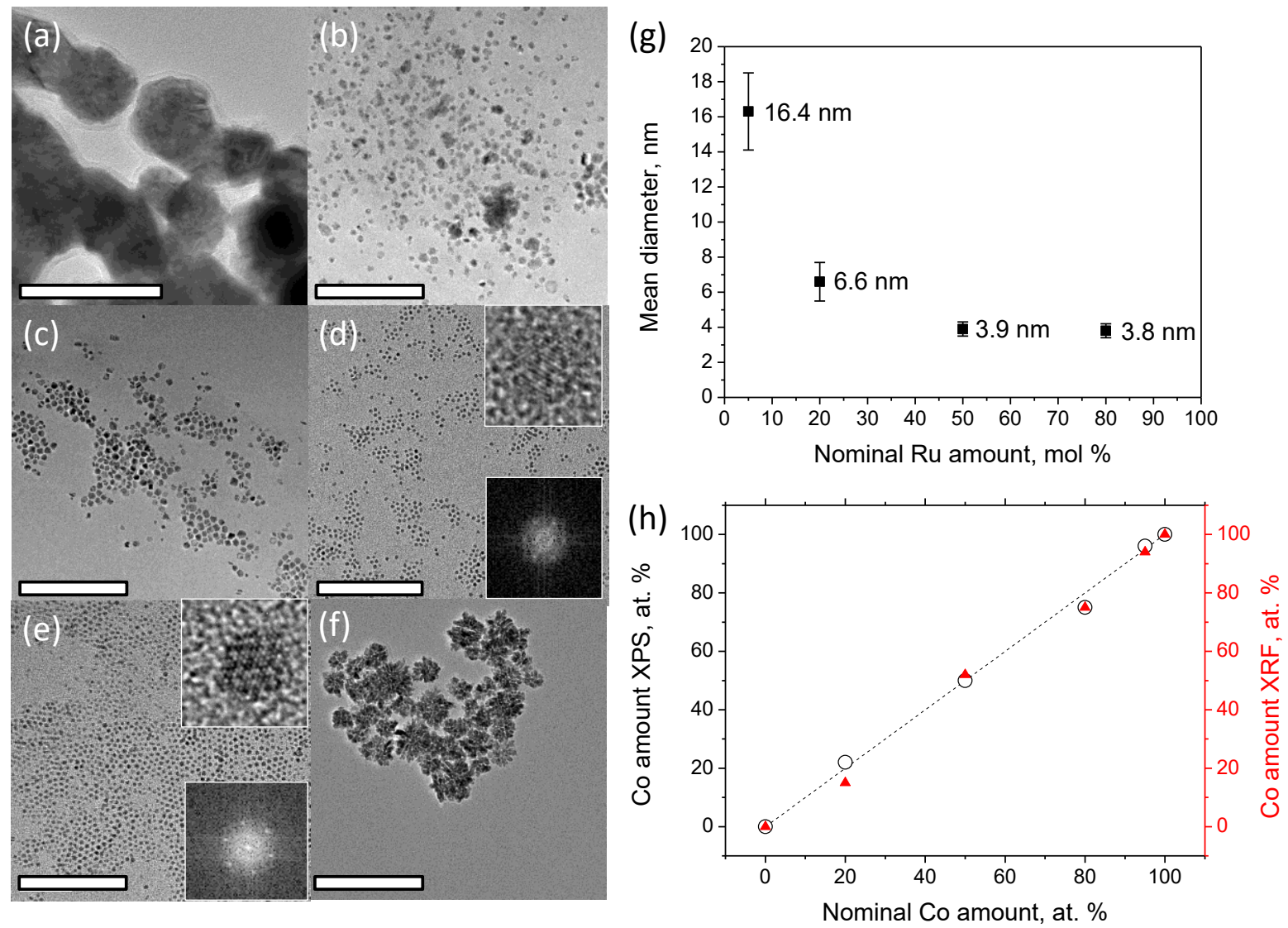

Figure 3. (a-f) TEM images of $\mathrm{Co}_{x} \mathrm{Ru}_{100-x}$ NPs (scale bar: $100 \mathrm{~nm}$ ), (g) mean size of the NPs as a function of the nominal Ru amount (at. \%) and (h) Co amounts (at. \%) measured by XPS and XRF as a function of the nominal Co amount (at. \%). The error bar in (g) corresponds to the standard deviation. Insets in (d) and (e): HRTEM images and corresponding fast Fourier transforms. (a): Co; (b): $\mathrm{CO}_{95} \mathrm{Ru}_{5}$; (c): $\mathrm{CO}_{80} \mathrm{Ru}_{20}$; (d): $\mathrm{CO}_{50} \mathrm{Ru}_{50}$; (e): $\mathrm{CO}_{20} \mathrm{Ru}_{80}$; (f): $\mathrm{Ru}$.

For each composition considered in this study, there is a very good agreement between the Co nominal amount and that determined by XRF or XPS (see Figure 3h). To probe the distribution of the metals in the particles, STEM-HAADF experiments coupled to 
EDX were undertaken. The results are presented in Figure 4. It can be clearly seen that, for the $\mathrm{CO}_{95} \mathrm{Ru}_{5}$ composition, the sample corresponds to Co-rich particles decorated by Ru tiny particles, indicating that, in this case, the two metals are not homogeneously distributed in the particles. The presence of $\mathrm{Ru}$ in this Co-rich core cannot however be excluded since a part of the Ru has probably helped to induce particle nucleation. This assertion is supported by the fact that the mean particle size of the $\mathrm{Co}_{95} \mathrm{Ru}_{5}$ particles is significantly lower than that of the pure Co particles. For the $\mathrm{Co}_{80} \mathrm{Ru}_{20}, \mathrm{Co}_{50} \mathrm{Ru}_{50}$ and $\mathrm{Co}_{20} \mathrm{Ru}_{80}$ samples, local EDX analyses show the formation of well-alloyed particles.

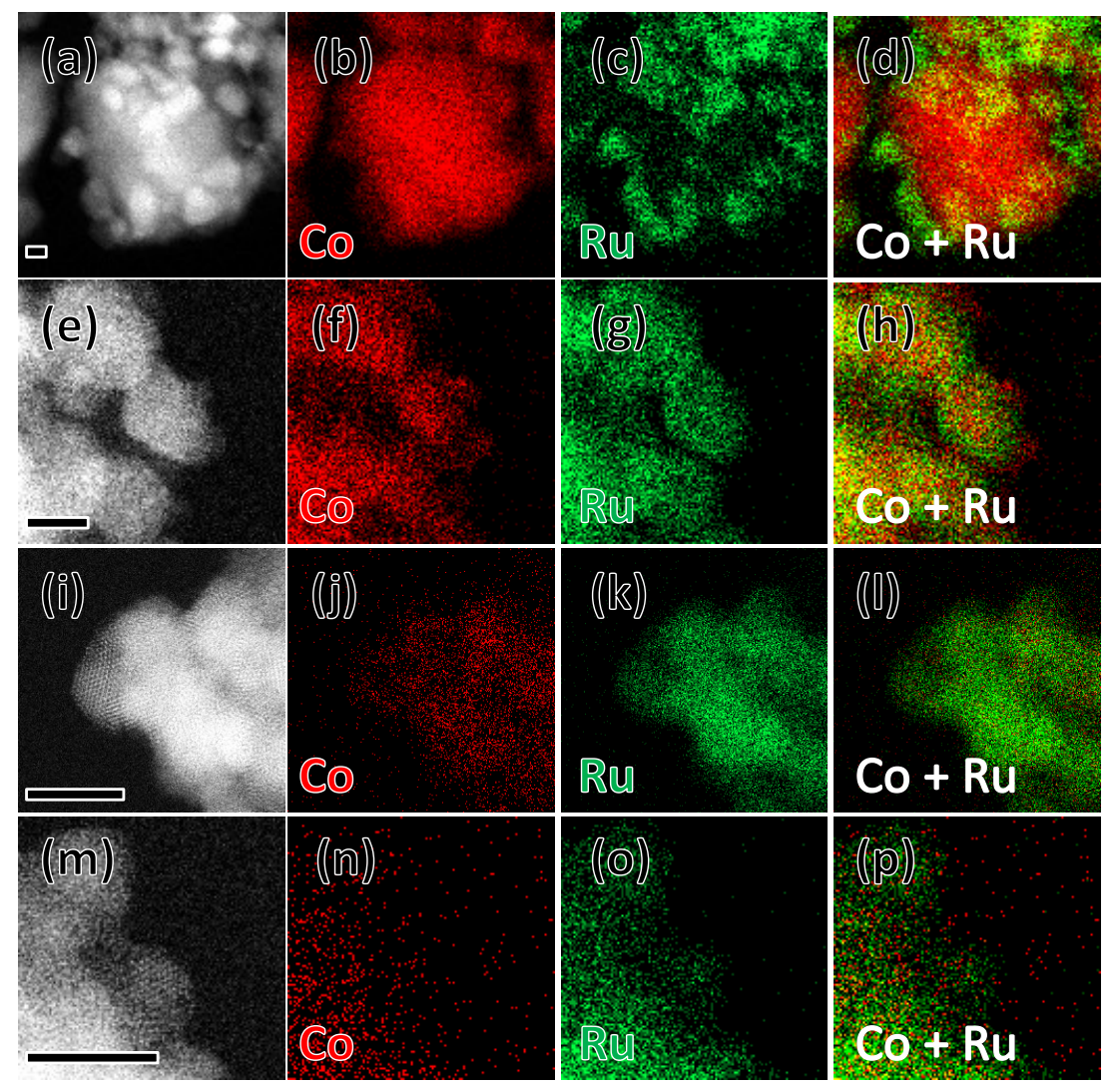

Figure 4. STEM-HAADF images $(a, e, i, m)$ and EDX elemental mapping of Co $(b, f, j, n), R u$ $(c, g, k, o)$ and Co+Ru overlap (d,h,l,p) for the $\mathrm{Co}_{x} \mathrm{Ru}_{100-x}$ samples. (a-d): $\mathrm{Co}_{95} \mathrm{Ru}_{5}$; (eh): $\mathrm{Co}_{80} \mathrm{Ru}_{20}$; (i-l): $\mathrm{CO}_{50} \mathrm{Ru}_{50} ;(\mathrm{m}-\mathrm{p}): \mathrm{Co}_{20} \mathrm{Ru}_{80}$. Scale bar: $5 \mathrm{~nm}$.

Similarly to $\mathrm{CO}_{80} \mathrm{Ru}_{20}$, XRD analyses were performed for all the compositions and the resulting X-ray diffraction patterns are presented in Figure 5. For pure Co sample, a mixture of mainly $h c p$ and $f c c$ phases is detected. Although hcp Co is normally the 
thermodynamically stable phase for Co at room temperature, the precipitation of both phases is frequently observed in the same sample at the nanometer scale with the fcc phase stabilized for small particle sizes. ${ }^{33}$ For the $\mathrm{CO}_{95} \mathrm{Ru}_{5}$ sample, the signal corresponds to the hcp phase of Co, while Ru cannot be clearly detected. This can be explained by the low amount of $\mathrm{Ru}$ in the sample and/or by very small coherent domain sizes, leading to broad peaks that are difficult to detect. This is in agreement with STEM-HAADF experiments coupled to EDX that reveal that the material consists of large Co-rich particles decorated by Ru NPs in the 5$10 \mathrm{~nm}$ size range (vide supra). For $\mathrm{CO}_{50} \mathrm{Ru}_{50}$ and $\mathrm{Co}_{20} \mathrm{Ru}_{80}$ samples, the $\mathrm{X}$-ray diffraction pattern is dominated by a very broad peak centered respectively at $49.8^{\circ}$ and $49.5^{\circ}$. This is explained by the small size of the NPs since HRTEM images show that the particles are very well crystallized (see Figure $3 \mathrm{~d}$ and e, insets). Finally, the pure Ru sample corresponds to a mixture of fcc and hcp phases (see Figure $\mathrm{S3}, \mathrm{SI}$ ).

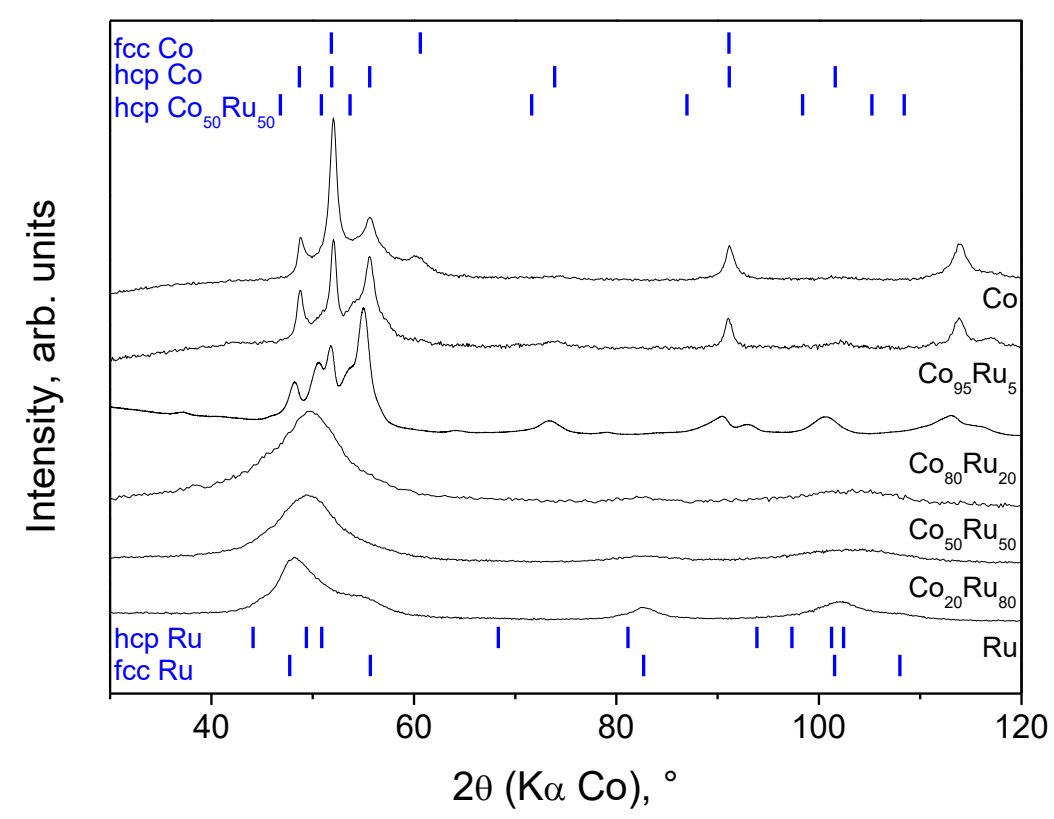

Figure 5. X-ray diffraction patterns for the $\mathrm{Co}_{x} \mathrm{Ru}_{100-\mathrm{x}}$ samples prepared in octan-1-ol.

Given that no ligand other than the acac moieties coming from the metal precursors is added during the synthesis, it can be assumed that the particles are stabilized against aggregation either by acac ligands, octan-1-ol and/or its oxidation products. Thermogravimetric and thermodifferential analyses coupled to IR and GC-MS spectroscopies 
were thus carried out in order to assess their amount and chemical nature (see Table 1 and Figure $\mathrm{S} 4$ for a representative example with $\mathrm{Co}_{80} \mathrm{Ru}_{20}$ ). Apart for pure $\mathrm{Co}$, a mass loss of about $10 \mathrm{wt} \%$, associated with an endothermic peak, is observed for all the materials and corresponds to the removal of the surface organic species. It compares well with what has been reported for Co nanorods. ${ }^{34}$ The lowest mass loss measured for pure Co particles prepared in octan-1-ol is explained by the low surface/volume ratio compared to the other particles prepared in this study. IR and GC-MS data show the formation of water and $\mathrm{CO}_{2}$ during the heating step and no decomposition fragments, showing that even with a very low $\mathrm{O}_{2}$ content (the analyses were performed in He atmosphere), the oxidative degradation of the organic surface species could not be avoided, possibly assisted by surface metal atoms.

Table 1. Physico-chemical characteristics and catalytic performances of the $\mathrm{Co}_{x} \mathrm{Ru}_{100-x}$ catalysts. $^{a}$

\begin{tabular}{|c|c|c|c|c|c|c|}
\hline Catalyst & $\begin{array}{l}S_{S A}{ }^{b} \\
m^{2} \cdot g^{-1}\end{array}$ & $\begin{array}{l}\text { Weight } \\
\text { loss TGA, } \\
\% \\
\end{array}$ & $\begin{array}{l}\text { Ligand } \\
\text { coverage, } \\
\mathrm{mg} \cdot \mathrm{m}^{-2}\end{array}$ & $\begin{array}{c}X(o l),{ }^{b} \\
\%\end{array}$ & $\begin{array}{c}\text { S(one), } \\
\%\end{array}$ & $\begin{array}{c}\mathrm{TON}^{\mathrm{c}} \\
\mathrm{mol}_{\text {alcohol }} \cdot \mathrm{mol}_{\text {surface metal }} \\
\text { atom }^{-1}\end{array}$ \\
\hline Co & $-d$ & 1.3 & $-{ }^{d}$ & 3 & $>99.9$ & $-d^{d}$ \\
\hline $\mathrm{CO}_{20} \mathrm{Ru}_{80}$ & 41.9 & 10.8 & 2.9 & 42 & $>99.9$ & 680 \\
\hline $\mathrm{CO}_{50} \mathrm{Ru}_{50}$ & 37.6 & 9.6 & 2.8 & 52 & $>99.9$ & 880 \\
\hline $\mathrm{Co}_{80} \mathrm{Ru}_{20}$ & 34.0 & 12.2 & 4.1 & 26 & $>99.9$ & 460 \\
\hline $\mathrm{Ru}$ & $-^{d}$ & 10.0 & $-{ }^{d}$ & 11 & $>99.9$ & $-d$ \\
\hline
\end{tabular}

${ }^{a}$ Reaction conditions: $( \pm)$-octan-2-ol $\left(0.95 \mathrm{~mol} \mathrm{~L}^{-1}\right)$, solvent $=$ decane, $\mathrm{T}=145{ }^{\circ} \mathrm{C}$, reaction time $=24 \mathrm{~h}$, inert atm., $\mathrm{n}_{\text {alcohol }} / \mathrm{n}_{\text {catalyst }}=100 \mathrm{~mol} / \mathrm{mol}$.

${ }^{\mathrm{b}} \mathrm{SSA}_{\mathrm{C}}$ stands for calculated specific surface area (see ESI), X(ol) for ( \pm )-octan-2-ol conversion and $\mathrm{S}(\mathrm{one})$ for ketone selectivity.

${ }^{\text {'T } T h e ~ a b s o l u t e ~ e r r o r ~ o n ~ T O N ~ i s ~ e s t i m a t e d ~ t o ~ b e ~} \pm 75 \mathrm{~mol} / \mathrm{mol}$

${ }^{\mathrm{d}}$ Not calculated. 
(a)

$\operatorname{Co} 2 p_{1 / 2} \quad$ Co $2 p_{3 / 2}$

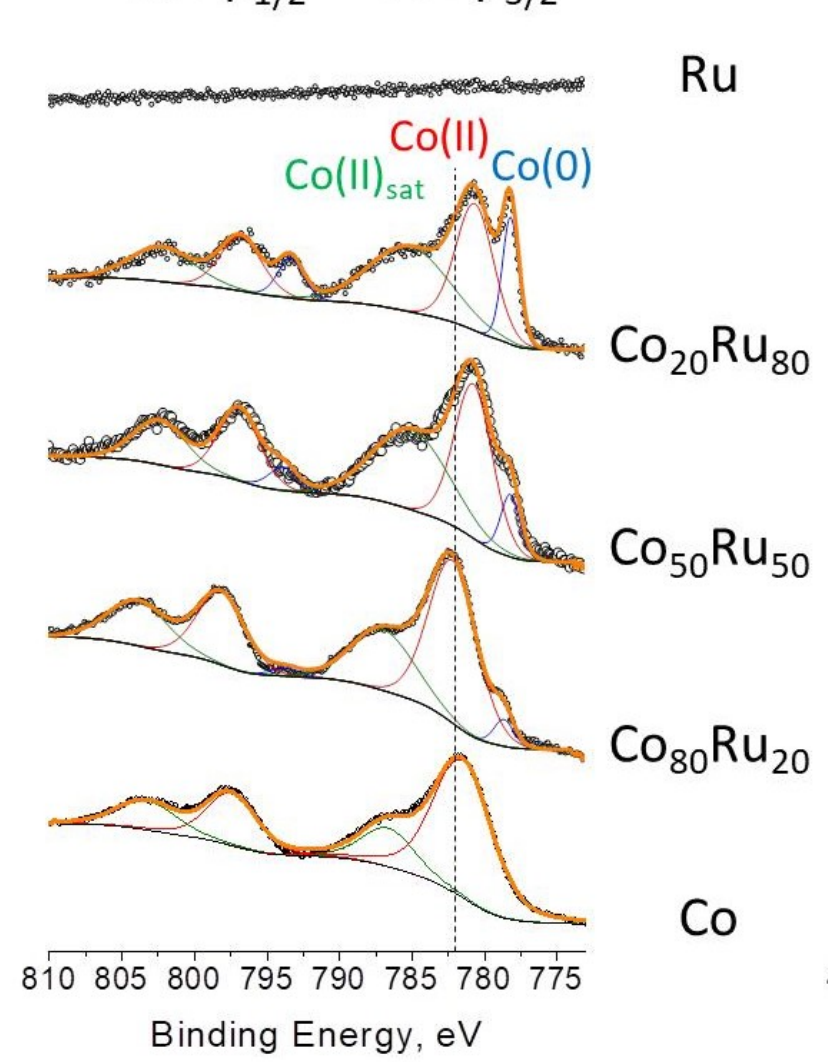

(b) $\quad \mathrm{Ru} 3 \mathrm{p}_{3 / 2}$

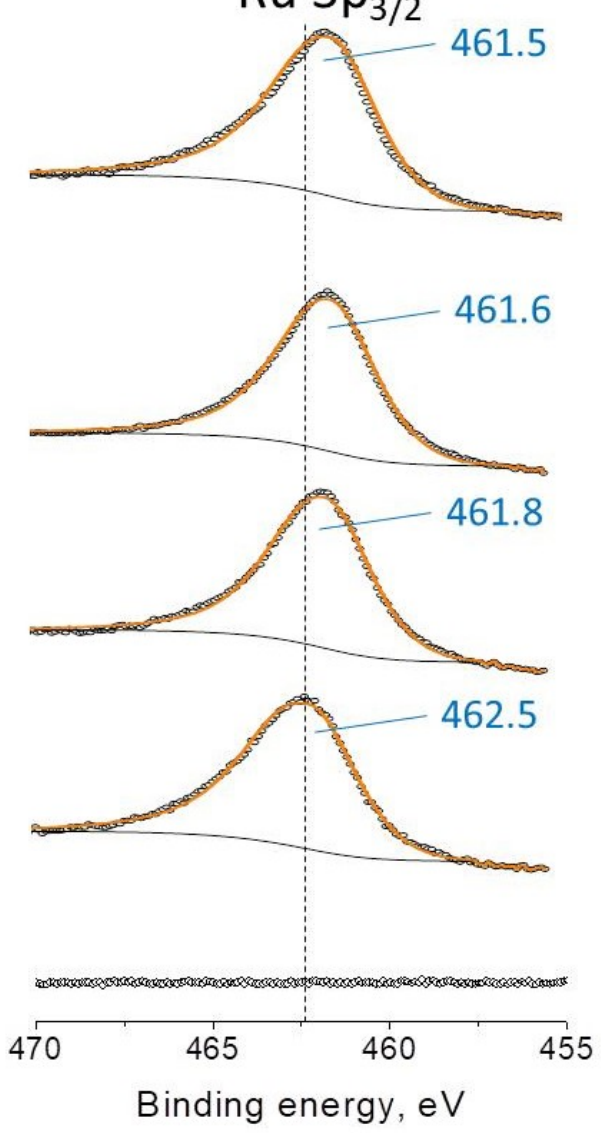

(c)

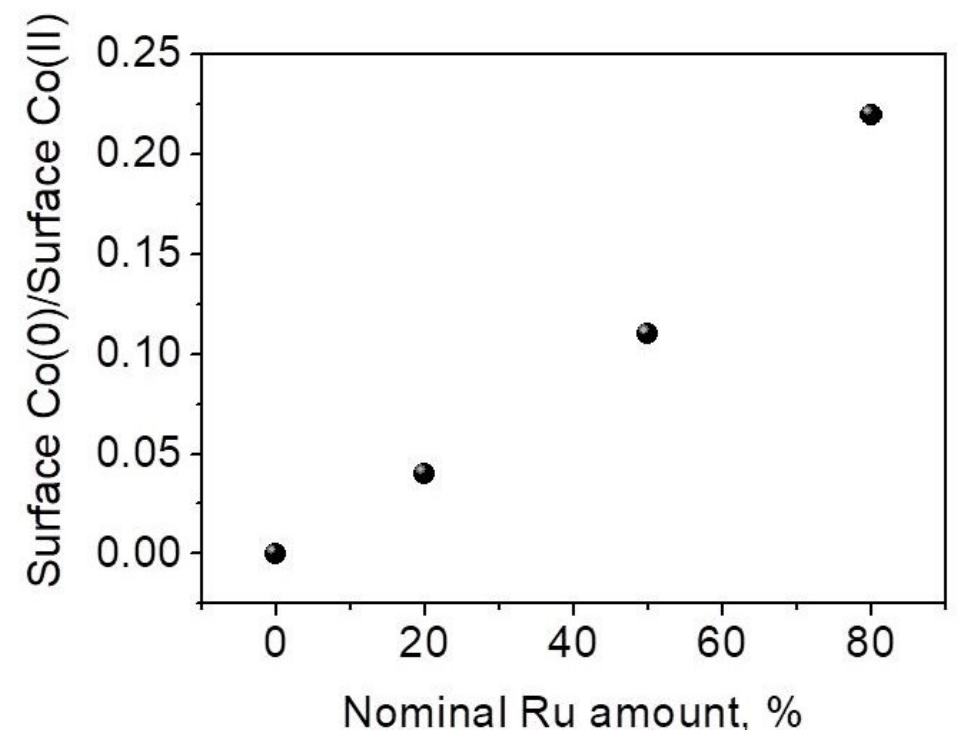

Figure 6. XPS high resolution Co $2 p$ (a) and $\mathrm{Ru} 3 p$ (b) spectra and (c) evolution of the surface $\mathrm{Co}(0) /$ surface $\mathrm{Co}(\mathrm{II})$ ratio vs. the nominal Ru amount. The circle symbols correspond to the measured data while the line in orange, black, blue, red and green correspond respectively to the calculated profile, the background and the peak contributions. 
The samples were further characterized by XPS. High resolution Co $2 p$ and Ru $3 p$ spectra are gathered in Figure $6 \mathrm{a}$ and $6 \mathrm{~b}$, respectively. For pure Co, only contributions corresponding to Co(II) species are detected while Co metal could not be detected, even if XRD evidenced clearly the formation of Co metal (see above). This is explained by the native CoO layer as well as the organic surface species that screens the metal core. With the incorporation of $\mathrm{Ru}$ in the material, characteristic peaks at $778.2 \mathrm{eV}$ and $793.2 \mathrm{eV}$ are detected, that are respectively assigned to the $2 p_{3 / 2}$ and $2 p_{1 / 2}$ components of Co metal. ${ }^{35} \mathrm{It}$ is noteworthy that the surface $\mathrm{Co}(0) /$ surface $\mathrm{Co}(\mathrm{II})$ ratio increases regularly with the $\mathrm{Ru}$ amount (Figure $6 \mathrm{c}$ ), suggesting that the introduction of $\mathrm{Ru}$ in the material promotes the reduction of cobalt, which is supported by previously results. ${ }^{9,12} \mathrm{~A}$ broad naturally asymmetric peak in the range $461.5-462.3 \mathrm{eV}$ is detected for all the Ru-containing samples. This peak is assigned to the Ru $3 p_{3 / 2}$ component, ${ }^{36}$ and its position is shifted monotonously to lower binding energies (BE) when increasing the Ru amount. Note that variations in peak positions for Co are also detected but no clear tendency can be seen. The decrease in Ru $3 p_{3 / 2}$ binding energy with the Ru amount can be interpreted as indicative of electron transfer processes between $\mathrm{Co}$ and $\mathrm{Ru},{ }^{13}$ suggesting intimate electronic Co-Ru interactions due to the formation of an alloy.

The elucidation of the mechanism accounting for the formation of the alloyed particles is beyond the scope of this study. Nevertheless, some interesting findings can be set out at this stage. First, the fact that the mean size dramatically decreases with increasing $\mathrm{Ru}$ amount (Figure 4g) suggests that this metal plays a prominent role in the size control of the bimetallic NPs. Since Ru is nobler than $\mathrm{Co}_{0}$, it is likely that Ru is reduced first and triggers Co nucleation. This has been recently exemplified in the formation of Co nanorods and platelets prepared by the polyol process in butane-1,2-diol where both Co(II) and Ru(III) precursors are added at the beginning of the synthesis, i.e. before the heating step. In these conditions, a tiny Ru seed could be evidenced in the exact center of the Co NP while the formation of a Co-Ru alloyed surface alloy could not be precluded. ${ }^{37}$ At this point, we can propose a two-step reaction mechanism: i) formation of tiny $\mathrm{Ru}$ seeds in the reaction medium allowing for the heterogeneous nucleation of $\mathrm{Co}$ and ii) solid-state diffusion of $\mathrm{Ru}$ and/or Co leading to the formation of a homogeneous alloy. Secondly, another important finding is that the formation of the alloy was only achieved in octan-1-ol while in BD, the two 
metals precipitated separately. It is well known that the strength of the reducing agent is an important parameter to control for the successful formation of the desired alloy. ${ }^{2}$ The reducing power of the two solvents used in this study can be appreciated using electrochemichal tools. Linear sweep voltammetry experiments were thus carried out with butane-1,2-diol and octan-1-ol at $25{ }^{\circ} \mathrm{C}$ and $60{ }^{\circ} \mathrm{C}$ (see Figure 7). At $25{ }^{\circ} \mathrm{C}$, the results show that the onset of oxidation is located at ca. $1.3 \mathrm{~V}$ vs. ECS for the two solvents. However, the oxidation current increases much more rapidly for butane-1,2-diol than for octan-1-ol for potentials higher than about $2.3 \mathrm{~V}$ vs. ECS, suggesting that on this Pt electrode, oxidation of $\mathrm{BD}$ is more pronounced than for octan-1-ol. At $60{ }^{\circ} \mathrm{C}$, the onset of the oxidation of the two solvents is shifted to lower potentials (respectively 1.1 and $0.8 \mathrm{~V}$ vs. ECS for octan-1-ol and $\mathrm{BD})$, as observed by Biacchi et al. ${ }^{39}$ The comparison of the currents measured at 25 and $60^{\circ} \mathrm{C}$ shows that oxidation is faster for butane-1,2-diol at high temperature than at room temperature while for octan-1-ol, the oxidation rate is not significantly impacted. One can note that a pre-peak is present for $B D$ at $60{ }^{\circ} \mathrm{C}$. This peak has been observed by others for EG but its origin is still not known and is beyond the scope of this study. ${ }^{38,39}$

(a)

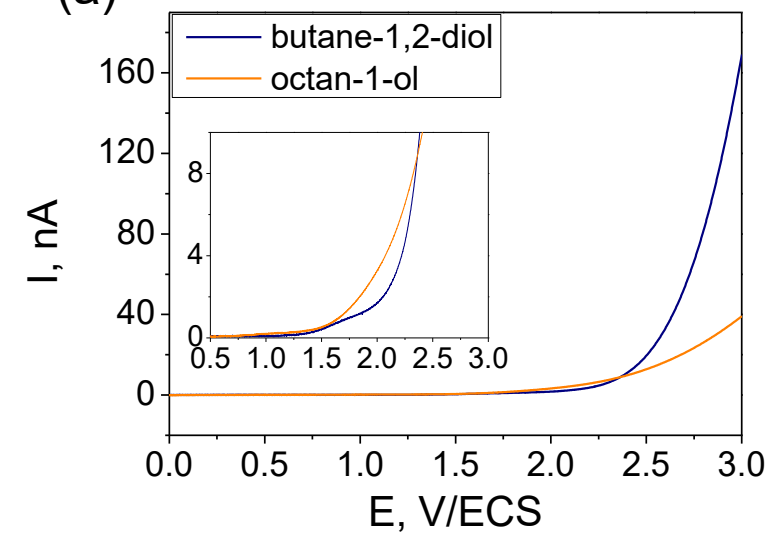

(b)

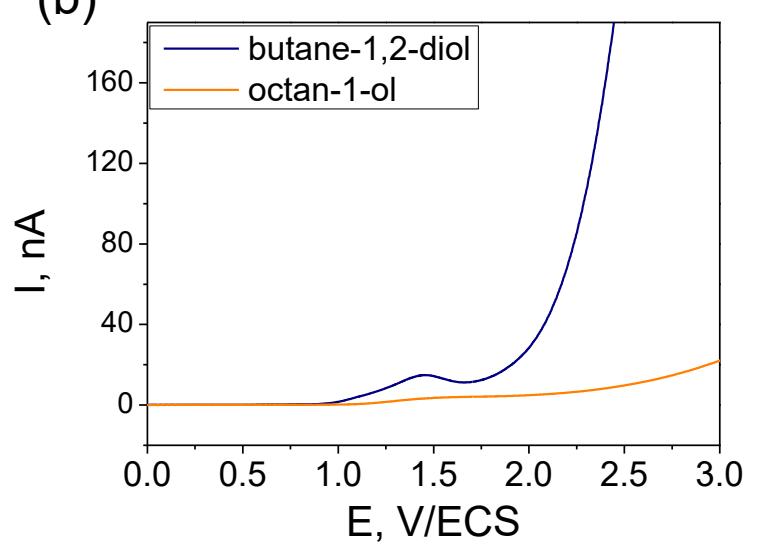

Figure 7. Linear sweep voltammograms corresponding to the oxidation of butane-1,2-diol and octan-1-ol at (a) $25^{\circ} \mathrm{C}$ (inset: magnified view) and (b) $60{ }^{\circ} \mathrm{C}$. See experimental part for details.

Although the potential value corresponding to the onset of solvent oxidation does not necessarily correspond to the thermodynamic value (overpotential may contribute), it is 
possible to conclude that BD is a stronger reducing agent than octan-1-ol, particularly at high temperature. This is in line with previous results: mono-alcohols are milder reducing agents than polyols. ${ }^{40}$ Since an increase in the reduction rate leads to an increase in supersaturation because of a higher number of metal atoms generated, ${ }^{41}$ it is expected that the highest supersaturation values will be obtained with BD rather than with octan-1-ol. Our results can thus tentatively be explained considering that the high supersaturation obtained with BD is detrimental for the formation of an alloy while the use of octan-1-ol is a way to obtain a better control of the nucleation step and may explain why this solvent is more suitable for the formation of the desired alloy.

\section{Catalytic acceptorless dehydrogenation of alcohols}

Compared to oxidative dehydrogenation of alcohols, acceptorless dehydrogenation of alcohols (ADA) is very attractive since no hydrogen acceptor, such as $\mathrm{O}_{2}$ or sacrificial carbonyl compounds or alkenes, are needed. Moreover, besides the desired carbonyl compound, molecular hydrogen, that is now considered as the fuel of the future, is generated and can be used for further hydrogenation processes. ${ }^{42}$ However, contrary to the oxidative dehydrogenation of alcohols, which is thermodynamically favored, ADA is very often endergonic and high temperatures are generally implemented to counterbalance the unfavorable $\Delta \mathrm{H}$ term by a favorable $-\mathrm{T} \cdot \Delta \mathrm{S}$ term. ${ }^{43}$

Since the $\mathrm{Co}_{x} \mathrm{Ru}_{100-x}$ particles are generated through reduction of $\mathrm{Co}(\mathrm{II})$ and $\mathrm{Ru}(\mathrm{III})$ species by octan-1-ol, it suggests that the latter is oxidized during the course of the reaction. Moreover, the as-formed particles can also catalyze the dehydrogenation of the remaining alcohol as was recently reported for Co particles prepared in butane-1,2-diol. ${ }^{37}$ GC-MS analyses were thus carried out on the liquid phase recovered from the synthesis of $\mathrm{CO}_{80} \mathrm{Ru}_{20}$ particles. They revealed the formation of octanal while $\mathrm{H}_{2}$ was evidenced in the gas phase together with air since no peculiar precaution was taken during sampling (see SI, Figure S5). However a very low alcohol conversion of $14 \%$ and a selectivity to octanal of $17 \%$ were found after $1 \mathrm{~h} 30$ at $190{ }^{\circ} \mathrm{C}$. As indicated below, this low selectivity is explained by the formation of C16 and C24 byproducts. 

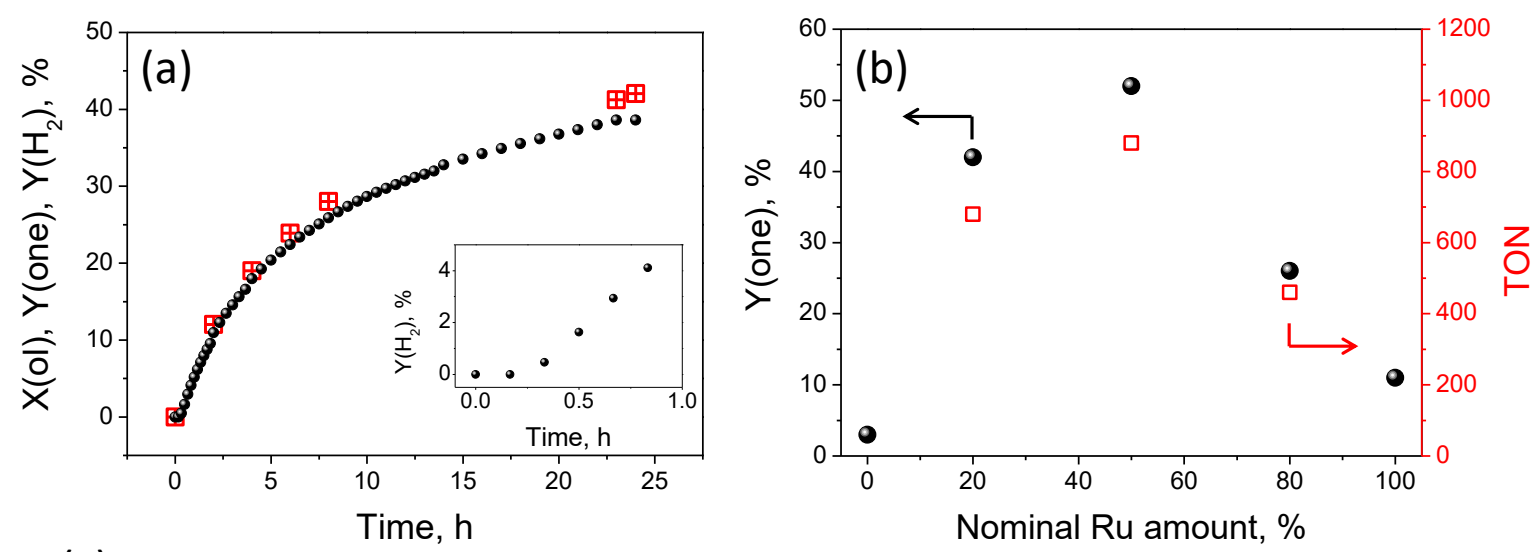

(c)

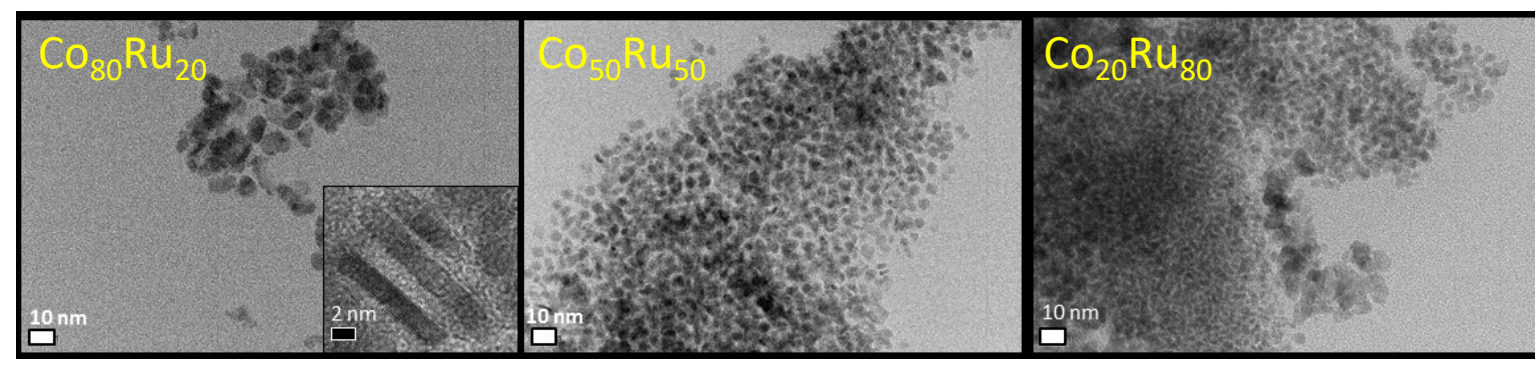

Figure 8. Catalytic test at $145{ }^{\circ} \mathrm{C}$ for $( \pm$ )-octan-2-ol dehydrogenation. (a) Conversion of ( \pm )octan-2-ol ( $\square)$, and yields in octan-2-one $(+)$ and $\mathrm{H}_{2}(\bullet)$ measured vs. time with the $\mathrm{Co}_{80} \mathrm{Ru}_{20}$ catalyst. A magnified view is given in the inset. (b) Measured yield in octan-2-one and TON for the $\mathrm{Co}_{x} \mathrm{Ru}_{100-x}$ nano-catalysts after $24 \mathrm{~h}$ reaction time at $145{ }^{\circ} \mathrm{C}$ (see ESI for details). (c) TEM images of the particles recovered after the catalytic tests.

The already formed particles were first tested for the acceptorless dehydrogenation of ( \pm )-octan-2-ol, which is considered as a model aliphatic alcohol ${ }^{44}$ harder to dehydrogenate compared to activated alcohols such as aromatic ones. ${ }^{45}$ The sample with the $\mathrm{Co}_{95} \mathrm{Ru}_{5}$ composition is excluded since it does not correspond to a well-defined alloy (vide supra). Results obtained for pure Co and Ru are also reported for comparison purposes (see Table 1). The evolution with time of the (t)-octan-2-ol conversion, $X(o l)$, the octan-2-one yield, $\mathrm{Y}$ (one) and $\mathrm{H}_{2}$ yield, $\mathrm{Y}\left(\mathrm{H}_{2}\right)$, are given for the $\mathrm{Co}_{80} \mathrm{Ru}_{20}$ catalyst as a representative example (see ESI, Figure S6 for the other compositions). Since an excellent selectivity (> 99.9\%) towards octan-2-one was evidenced, the ketone yield is equal to the alcohol conversion. $\mathrm{Y}$ (one) increases regularly with time to reach $42 \%$ after $24 \mathrm{~h}$ at $145^{\circ} \mathrm{C}$. A closer inspection at low reaction times (see Figure $8 \mathrm{a}$, inset) shows that there is an induction period (about 20 $\min$ ) before the catalyst can effectively catalyze the dehydrogenation of the alcohol. This is 
explained by the time necessary for the in situ reduction of the native oxide layer to yield the active metal surface.

All the catalysts were found to be active towards the dehydrogenation of ( \pm )-octan-2ol but different ketone yields were measured depending on the catalyst composition. There is always a good agreement between $Y($ one $)$ and $Y\left(H_{2}\right)$ showing that the reaction follows the acceptorless dehydrogenation pathway. A clear synergetic effect between $\mathrm{Co}$ and $\mathrm{Ru}$ is observed in Figure 8b since higher yields are measured for the nanoalloys than for pure Co, that is almost inactive, and pure Ru. Several reasons can be invoked to explain these differences: i) the nature and/or the amount of surface ligands, ii) the Co/Ru molar ratio iii) the specific surface area of the catalyst and iv) the nature and the relative proportions of the exposed crystal facets. The acceptorless dehydrogenation of alcohols is generally considered a structure sensitive reaction, as was recently reported for pristine $\mathrm{Co}^{24}$ and $\mathrm{Cu}^{46}$ based on DFT calculations. Thus, playing on the size and the shape of the particles is a way to modulate the catalytic activity. Nevertheless, this structure sensitivity can be altered by the presence of surface ligands. ${ }^{47}$ The very low activity observed for the pure Co catalyst is probably explained by its morphology corresponding to ill-defined aggregated particles, hence by a significantly lower specific surface area than the other catalysts considered in this study, rather than surface passivation. Indeed, all the $\mathrm{Co}_{x} \mathrm{Ru}_{100-x}$ particles were prepared with the same metal precursors under identical conditions and only the composition was varied. Consequently, it is likely that similar surface species (i.e. acac moieties, octan-1-ol and/or its oxidation products), are adsorbed onto the different crystal facets. For Ru particles, the low activity $\left(\mathrm{Y}(\mathrm{one})=11 \%\right.$ after $24 \mathrm{~h}$ at $\left.145^{\circ} \mathrm{C}\right)$ is probably explained, at least partially, by a partial aggregation, hence reducing the available metal surface.

Because the catalysts do not display the same specific surface areas, it is preferable to compare catalytic activity based on turnover numbers (TONs). Since TON calculations are based on geometrical considerations in this work (see experimental part), only particles displaying a well-defined morphology were considered, namely the bimetallic samples that correspond to hexagonal platelets, i.e. $\mathrm{Co}_{80} \mathrm{Ru}_{20}, \mathrm{CO}_{50} \mathrm{Ru}_{50}$ and $\mathrm{Co}_{20} \mathrm{Ru}_{80}$. The results (Table 1 and Figure $8 \mathrm{~b}$ ) show that the $\mathrm{CO}_{50} \mathrm{Ru}_{50}$ catalyst is the most active one with the highest TON value of 880 , while $\mathrm{CO}_{80} \mathrm{Ru}_{20}$ and $\mathrm{CO}_{20} \mathrm{Ru}_{80}$ catalysts exhibit somewhat lower TON values, respectively 680 and 460 (see Table 1). In a previous study with Co particles, we have shown 
that a surface coverage by organic species higher than ca. $4 \mathrm{mg} \cdot \mathrm{m}^{-2}$ could be detrimental to catalysis. ${ }^{48}$ This can explain, to some extent, the lowest TON value determined for the $\mathrm{CO}_{20} \mathrm{Ru}_{80}$ catalyst that has a ligand coverage of $4.1 \mathrm{mg} \cdot \mathrm{m}^{-2}$. It is noticeable that the TON value of 880 measured with $\mathrm{CO}_{50} \mathrm{Ru}_{50}$ NPs is significantly higher than that reported for Co hexagonal platelets prepared with the polyol process and stabilized by laurate (TON $=600$ ) or palmitate $\left(\right.$ TON $=430$ ) ligands under identical catalytic conditions. ${ }^{47}$ It is also higher than the TON reported for Co platelets prepared with the organometallic route where no activity could be measured with, again, the same reaction conditions. ${ }^{48}$ However, in the last case, the observed inactivity was explained by the presence of chlorine atoms adsorbed onto the metal surface that suppressed the activity, emphasizing the importance of the nature of the surface species that stabilize the NPs. The particles were recovered after catalysis to assess a possible evolution of the morphology. The platelet shape is maintained for the $\mathrm{Co}_{80} \mathrm{Ru}_{20}$ and $\mathrm{CO}_{50} \mathrm{Ru}_{50}$ catalysts (see Figure $8 \mathrm{c}$ ) with no peculiar aggregation of the particles. However, for $\mathrm{CO}_{20} \mathrm{Ru}_{80}$, important shape modifications are obtained: besides the small platelets that were initially present before the test, larger particles can be observed, suggesting that dissolutionreprecipitation mechanisms have occurred during catalysis.
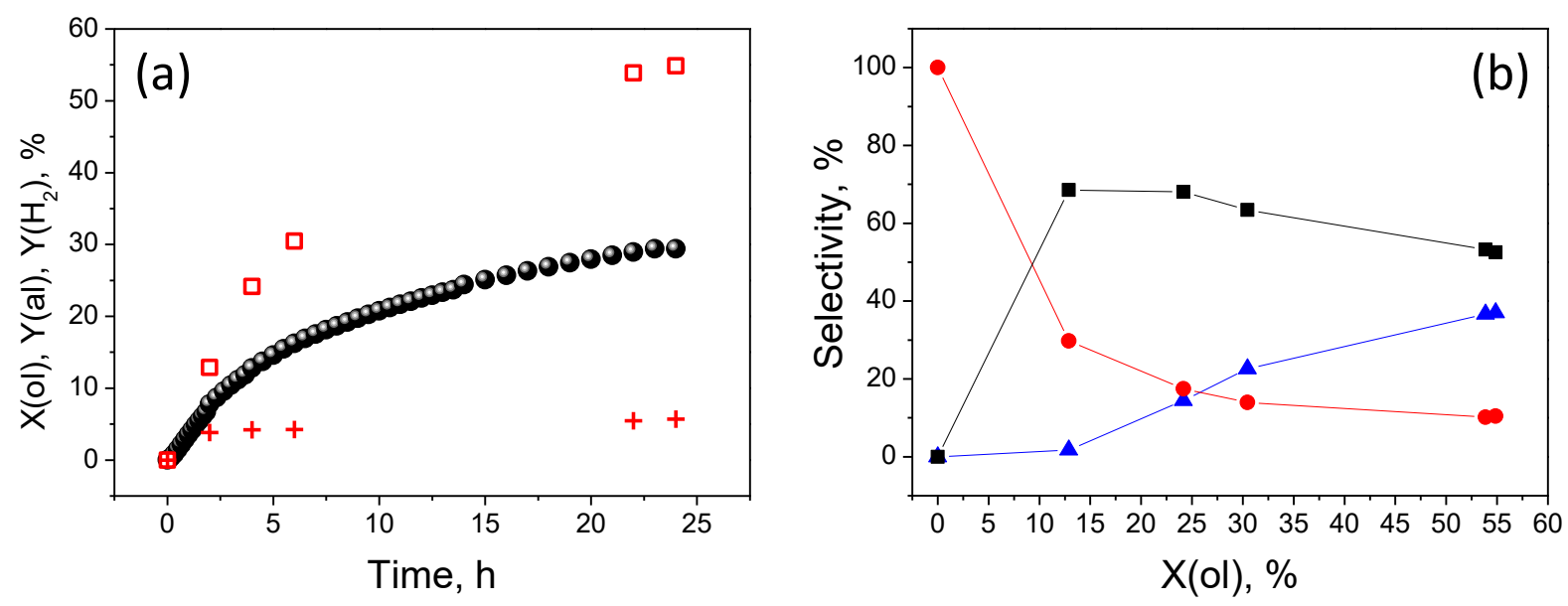

Figure 9. Catalytic test at $145^{\circ} \mathrm{C}$ for octan-1-ol dehydrogenation. (a) Conversion of octan1-ol ( $\square$ ), and yields in octanal (+) and $\mathrm{H}_{2}(\bullet)$ measured vs. time with the $\mathrm{CO}_{50} \mathrm{Ru}_{50}$ catalyst. (b) Selectivity in octanal $(\bullet), C 16(\boldsymbol{\square})$ and C24 (A) products vs. octan-1ol conversion, $\mathrm{X}(\mathrm{ol})$ (see text for details). 
The nanocatalyst with the $\mathrm{CO}_{50} \mathrm{Ru}_{50}$ composition, being the most active in the dehydrogenation of octan-2-ol, was chosen to assess its activity towards the dehydrogenation of octan-1-ol (see Figure 9a). Very interestingly, unsupported $\mathrm{CO}_{50} \mathrm{Ru}_{50}$ nanoalloys are found to be much more active than heterogeneous catalysts corresponding to $\mathrm{Co}$, or Ru supported on various supports tested under identical conditions. ${ }^{49,50}$ An octan1-ol conversion of $55 \%$ was found after $24 \mathrm{~h}$ at $145^{\circ} \mathrm{C}$ for the nanoalloys, to be compared to $10 \%$ obtained with $\mathrm{Co} / \mathrm{TiO}_{2}{ }^{49}$ or $8 \%$ with $\mathrm{Ru} / \mathrm{TiO}_{2},{ }^{50}$ tested under the same conditions. However, a very low octanal yield of $6 \%$ was measured after $24 \mathrm{~h}$ for the nanocatalyst, which is explained by a poor selectivity to octanal of $10 \%$. Indeed, besides the aldehyde, the formation of C16 and C24 by-products was evidenced. These compounds originate from aldol condensation and further hydrogenation processes. ${ }^{49,50}$ This also explains why the octan-1-ol conversion (55\%) is much higher than the yield of $\mathrm{H}_{2}(29 \%$ after $24 \mathrm{~h}$, see Figure 9a); with an acceptorless mechanism, two similar values are expected. To explain our results, it is assumed that $\mathrm{H}_{2}$ generated by dehydrogenation of alcohols is next used to hydrogenate aldol condensation products following a reaction pathway that has been proposed previously with $\mathrm{Cu} / \mathrm{ZrO}_{2}$ catalyst. ${ }^{50}$ The evolution of the selectivity as a function of the octan1-ol conversion is presented in Figure 9b. One can see that the selectivity to octanal decreases when the conversion increases. The selectivity to C16 byproducts increases with the conversion before decreasing and reaching a plateau. For a conversion higher than ca. 10 $\%$, C24 products start to be detected and their proportion increases with the conversion to reach $37 \%$ after $24 \mathrm{~h}$ at $145^{\circ} \mathrm{C}$. Although the selectivity to octanal has to be improved, these results are very encouraging since pure Co particles prepared by the polyol process were not active towards octan-1-ol dehydrogenation: the formation of CoRu nano-alloys appears as an efficient way to modify the chemoselectivity of these unsupported nanocatalysts.

\section{Conclusion}

By carefully controlling the co-reduction conditions of $\mathrm{Co}$ and $\mathrm{Ru}, \mathrm{Co}_{x} \mathrm{Ru}_{100-x}$ particles have been prepared in octan-1-ol by a simple soft chemistry method, using common metal salts without the need of additional stabilizing ligands that are usually necessary to avoid particle aggregation. All the different compositions considered in this work were proved to be alloys, except the $\mathrm{Co}_{95} \mathrm{Ru}_{5}$ sample where phase segregation is observed. Even if there is a very good 
agreement between the initial and measured Co/Ru molar ratio, local analyses showed that the composition may vary from one particle to another. However, inside a single particle, there is a homogeneous distribution of Ru and Co elements as proved by line scan analyses. The particles were tested for the acceptorless dehydrogenation of ( \pm )-octan-2-ol and octan1-ol. With the secondary alcohol, pure Co and Ru particles were almost inactive but for the nanoalloys, the secondary alcohol was converted into the corresponding ketone with an excellent selectivity. A clear synergetic effect was observed, with the $\mathrm{Co}_{50} \mathrm{Ru}_{50}$ composition being the most active catalyst. This catalyst was also tested for the dehydrogenation of octan-1-ol. A primary alcohol conversion higher than that obtained with the secondary alcohol was measured but at the expense of octanal selectivity and C16 and C24 condensation products were identified. Further work will be devoted to the elucidation of the reaction mechanism and to a more complete evaluation of the catalytic properties of these nanoalloys, not only for acceptorless alcohol dehydrogenation but also for other valuable reactions such as the amination of alcohols.

\section{Author contributions}

Brandon Azeredo: investigation (materials synthesis), validation; Tayssir Ben Ghzaiel: investigation (materials synthesis), validation; Ning Huang: Investigation (materials synthesis), validation; Kamila Kaźmierczak: investigation (catalytic tests), formal analysis, validation; Wenjie Shen: investigation (TEM studies), writing - review and editing; Guillaume Wang: Investigation (TEM studies); Delphine Schaming: investigation (electrochemical studies); Patricia Beaunier: investigation (TEM studies); Philippe Decorse: investigation (XPS analyses) Noémie Perret: supervision, writing - review and editing; Jennifer Peron: writing review and editing; Marion Giraud: writing - review and editing; Carine Michel: supervision; Lorette Sicard: supervision, writing - original draft; Jean-Yves Piquemal: supervision, writing - original draft, funding acquisition.

\section{Conflicts of interest}

The authors declare no competing interests. 


\section{Acknowledgements}

The work was done as a part of the ANR TANOPOL project (ANR-15-CE07-0011-01). Ning Huang would like to thank the Chinese Scolarship Council. The authors are grateful to Prof. Christian Ricolleau for fruitful discussions on electron diffraction, to Dr. Sophie Nowak for XRF analyses and to Alexandre Chevillot for thermal analyses. XPS equipment was funded by the Région Île-de-France, convention SESAME n¹6016303 and the Labex SEAM (Science and Engineering for Advanced Materials and devices).

\section{Graphical table of contents}

\section{$\mathrm{Co}_{x} \mathrm{Ru}_{100-x}$ nano-alloys}

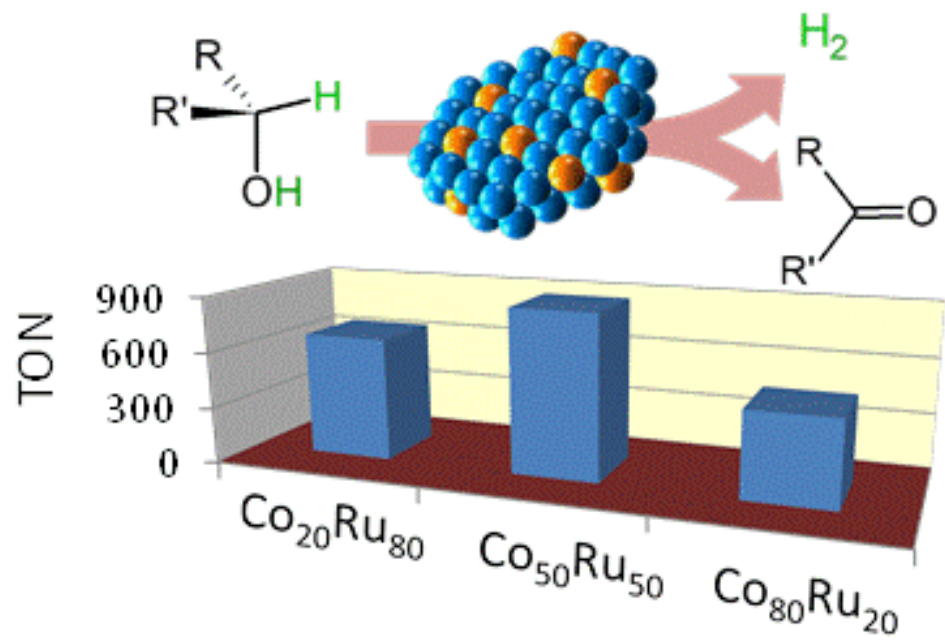

\section{References}

1 D. Alloyeau, C. Mottet and C. Ricolleau, Eds., Nanoalloys, Synthesis, Structure and Properties, Springer-Verlag, London, 2012.

2 K. D. Gilroy, A. Ruditskiy, H. C. Peng, D. Qin and Y. Xia, Chem. Rev., 2016, 116, 1041410472.

3 S. Shan, J. Luo, L. Yang and C. J. Zhong, Catal. Sci. Technol., 2014, 4, 3570-3588.

4 H. Fang, J. Yang, M. Wen and Q. Wu, Adv. Mater., 2018, 30, 1-10.

5 E. Iglesia, S. L. Soled, R. A. Fiato and G. H. Via, J. Catal., 1993, 143, 345-368.

6 M. Reinikainen, M. K. Niemelä, N. Kakuta and S. Suhonen, Appl. Catal. A Gen., 1998, 174, 61-75. 
7 A. Tavasoli, R. M. Malek Abbaslou and A. K. Dalai, Appl. Catal. A Gen., 2008, 346, 5864.

8 J. Hong, E. Marceau, A. Y. Khodakov, L. Gaberová, A. Griboval-Constant, J. S. Girardon, C. La Fontaine and V. Briois, ACS Catal., 2015, 5, 1273-1282.

9 Y. Wang, Y. Lu, Q. Cao and W. Fang, Chem. Commun., 2020, 56, 3765-3768.

10 Y. Jin, F. Chen and J. Wang, ACS Sustain. Chem. Eng., 2020, 8, 2783-2792.

11 G. P. Rachiero, U. B. Demirci and P. Miele, Int. J. Hydrogen Energy, 2011, 36, 70517065.

12 H. Wang, C. Gao, R. Li, Z. Peng, J. Yang, J. Gao, Y. Yang, S. Li, B. Li and Z. Liu, ACS Sustain. Chem. Eng., 2019, 7, 18744-18752.

13 W. Li, Y. Zhao, Y. Liu, M. Sun, G. I. N. Waterhouse, B. Huang, K. Zhang, T. Zhang and S. Lu, Angew. Chemie - Int. Ed., 2021, 60, 3290-3298.

14 Y. Zhu, S. Zhang, Y. Ye, X. Zhang, L. Wang, W. Zhu, F. Cheng and F. Tao, ACS Catal., 2012, 2, 2403-2408.

15 D. Gao, H. Li and X. Cheng, ECS Trans., 2015, 66, 57-68.

16 C. Gao, H. Wang, S. Li, B. Liu, J. Yang, J. Gao, Z. Peng, Z. Zhang and Z. Liu, Electrochim. Acta, 327, 134958.

17 Q. Yang, Y. Cui, Q. Li, J. Cai, D. Wang and L. Feng, ACS Sustain. Chem. Eng., 2020, 8, 12089-12099.

18 J. Su, Y. Yang, G. Xia, J. Chen, P. Jiang and Q. Chen, Nat. Commun., 2017, 8, 1-10.

19 J. V Medina-Flores, A. Manzo-Robledo, J. M. Mora-Hernández and E. M. Arce Estrada, Int. J. Hydrogen Energy, 2017, 42, 38-45.

20 Y. Xu, Y. Li, S. Yin, H. Yu, H. Xue, X. Li, H. Wang and L. Wang, Nanotechnology, 2018, 29, 225403.

21 Z. Wei, Y. Liu, Z. Peng, H. Song, Z. Liu, B. Liu, B. Li, B. Yang and S. Lu, ACS Sustain. Chem. Eng., 2019, 7, 7014-7023.

22 S. B. Qadri, T. M. Keller, M. Laskoski, C. A. Little, M. S. Osofsky and H. R. Khan, Appl. Phys. Lett., 91, 2005-2008.

23 D. Zitoun, C. Amiens, B. Chaudret, M.-C. Fromen, P. Lecante, M.-J. Casanove and M. Respaud, J. Phys. Chem. B, 2003, 107, 6997-7005.

24 A. Viola, J. Peron, K. Kazmierczak, M. Giraud, C. Michel, L. Sicard, N. Perret and P. Beaunier, Catal. Sci. Technol., 2018, 8, 562-572.

25 A. Viola, M. Peboscq, J. Peron, M. Giraud, L. Sicard, R. K. Ramamoorthy, B. Azeredo, S. Nowak, P. Decorse, G. Viau and J.-Y. Piquemal, Catal. Today, 2019, 333, 97-104. 
26 L. Lutterotti, D. Chateigner, S. Ferrari and J. Ricote, Thin Solid Films, 2004, 450, 34-41.

27 T. B. Massalski, Ed., Binary alloy phase diagrams. Vol. 1, ASM International, 1986.

28 F. Fiévet, S. Ammar-Merah, R. Brayner, F. Chau, M. Giraud, F. Mammeri, J. Peron, J.-Y. Piquemal, L. Sicard and G. Viau, Chem. Soc. Rev., 2018, 47, 5187-5233.

29 D. Alloyeau, G. Prévot, Y. Le Bouar, T. Oikawa, C. Langlois, A. Loiseau and C. Ricolleau, Phys. Rev. Lett., 2010, 105, 1-4.

30 A. M. Ruppert, M. Jędrzejczyk, N. Potrzebowska, K. Kaźmierczak, M. Brzezińska, O. Sneka-Płatek, P. Sautet, N. Keller, C. Michel and J. Grams, Catal. Sci. Technol., 2018, 8, 4318-4331.

31 M. Di Vece, S. Bals, J. Verbeeck, P. Lievens and G. Van Tendeloo, Phys. Rev. B Condens. Matter Mater. Phys., 2009, 80, 1-4.

32 K. Pelzer, O. Vidoni, K. Philippot, B. Chaudret and V. Collière, Adv. Funct. Mater., 2003, 13, 118-126.

33 O. Kitakami, H. Sato, Y. Shimada, F. Sato and M. Tanaka, Phys. Rev. B - Condens. Matter Mater. Phys., 1997, 56, 13849-13854.

34 K. Aït Atmane, F. Zighem, Y. Soumare, M. Ibrahim, R. Boubekri, T. Maurer, J. Margueritat, J.-Y. Piquemal, F. Ott, G. Chaboussant, F. Schoenstein, N. Jouini and G. Viau, J. Solid State Chem., 2013, 197, 297-303.

35 M. C. Biesinger, B. P. Payne, A. P. Grosvenor, L. W. M. Lau, A. R. Gerson and R. S. C. Smart, Appl. Surf. Sci., 2011, 257, 2717-2730.

36 D. J. Morgan, Surf. Interface Anal., 2015, 47, 1072-1079.

37 R. K. Ramamoorthy, A. Viola, B. Grindi, J. Peron, C. Gatel, M. Hytch, R. Arenal, L. Sicard, M. Giraud, J.-Y. Piquemal and G. Viau, Nano Lett., 2019, 19, 9160-9169.

38 F. Bonet, C. Guéry, D. Guyomard, R. Herrera Urbina, K. Tekaia-Elhsissen and J. M. Tarascon, Solid State Ionics, 1999, 126, 337-348.

39 A. J. Biacchi and R. E. Schaak, ACS Nano, 2011, 5, 8089-8099.

40 M. Ishijima, J. L. Cuya Huaman, H. Wakizaka, K. Suzuki, H. Miyamura and J. Balachandran, Inorg. Chem., 2021, 60, 14436-14445.

41 H. X. Lin, Z. C. Lei, Z. Y. Jiang, C. P. Hou, D. Y. Liu, M. M. Xu, Z. Q. Tian and Z. X. Xie, J. Am. Chem. Soc., 2013, 135, 9311-9314.

42 C. Gunanathan and D. Milstein, Science, 2013, 341, 1229712.

43 R. H. Crabtree, Chem. Rev., 2017, 117, 9228-9246.

44 S. E. Davis, M. S. Ide and R. J. Davis, Green Chem., 2013, 15, 17-45.

45 K. I. Shimizu, K. Kon, M. Seto, K. Shimura, H. Yamazaki and J. N. Kondo, Green Chem., 
$2013,15,418-424$.

46 Z. T. Wang, Y. Xu, M. El-Soda, F. R. Lucci, R. J. Madix, C. M. Friend and E. C. H. Sykes, J. Phys. Chem. C, 2017, 121, 12800-12806.

47 K. Kaźmierczak, R. K. Ramamoorthy, A. Moisset, G. Viau, A. Viola, M. Giraud, J. Peron, L. Sicard, J. Y. Piquemal, M. Besson, N. Perret and C. Michel, Catal. Sci. Technol., 2020, 10, 4923-4937.

48 K. Kaźmierczak, D. Yi, A. Jaud, P. F. Fazzini, M. Estrader, G. Viau, P. Decorse, J. Y. Piquemal, C. Michel, M. Besson, K. Soulantica and N. Perret, J. Phys. Chem. C, 2021, 125, 7711-7720.

49 K. Kaźmierczak, C. Pinel, S. Loridant, M. Besson, C. Michel and N. Perret, Chempluschem, 2020, 85, 1315-1324.

50 K. Kaźmierczak, A. Salisu, C. Pinel, M. Besson, C. Michel and N. Perret, Catal. Commun., 2021, 148, 106179. 